\title{
ESTUDIO TEÓRICO Y EXPERIMENTAL DE UN PROTOTIPO DE INVERNADERO BASADO EN UNA ESTRUCTURA RESISTENTE PLANA, LIGERA, ELÁSTICA Y ALTAMENTE DEFORMABLE
}

\author{
( THEORETICAL AND EXPERIMENTAL STUDY OF AN GREEN HOUSE PROTOTYPE BASED ON \\ A PLANE, LIGHT, ELASTIC AND HIGHLY DEFORMED RESISTENT STRUCTURE)
}

José L.de la Cruz Fernández*, Dr. Ingeniero Agrónomo ETS Ingenieros Agrónomos y Montes. Univ. de Córdoba

ESPAÑA
Directores de Tesis:

José A. Gistas Peyrona, Dr. Ingeniero Industrial

Antonio López Pinto, Dr. Ingeniero Agrónomo

Fecha de recepción: 5-VIII-96

\section{RESUMEN}

El creciente empleo de estructuras ligeras, tanto en invernaderos en países de clima templado, como, más recientemente, en arquitectura textil para instalaciones de recreo, justifica el desarrollo de este tipo de estructuras.

El objeto del presente trabajo es contribuir con un nuevo modelo de estructura de cubierta ligera y flexible, aportando un modelo teórico que se presenta, tras su validación con datos experimentales obtenidos sobre un prototipo, como una metodología adecuada para el cálculo de dicha estructura. La obtención de conclusiones sobre el comportamiento estructural y el estudio de costes realizado sobre el prototipo permiten, dada su viabilidad técnica y económica, proponerlo como una solución alternativa a los invernaderos tradicionales.

\section{SUMMMARY}

The development of light structures is justified because of its increasing use for greenhouses in temperate climate countries as is the creation of a new concept of textile architecture for recreational buildings.

The aim of this work is to present a new model for a flexible and light roofing structure. The model has been validatet with data obtained from a prototype, and so it is considered a suitable methology for the calculation of these structures. The conclusions on its structural behaviour as its technical and economic feasibility allow this sort of structures to be proposed as an alternative to traditional greenhouses.

\section{INTRODUCCIÓN}

En el sector del cultivo protegido se observan très grandes líneas de evolución de esta tecnología, en función del grado de protección de los cultivos.

Una primera línea de evolución sería la constituida por los invernaderos de estructura y cubierta de materiales

* Tesis leída el 21 de marzo de 1995 en la ETSIAM de Córdoba. Obtuvo la calificación de Apto Cum Laude. rígidos. Sobre este invernadero se han incorporado en los últimos tiempos mejoras en su esqueleto estructural: utilización de acero inoxidable y aluminio anodizado en los herrajes para recibir el material de cerramiento (vidrio o placa,...). Debido a su alto coste de inversión, este tipo de estructuras se utiliza en zonas frías o se construye para el cultivo de plantas de alto valor, para investigación o para jardines botánicos.

En segundo lugar, en zonas templadas se desarrollan invernaderos que utilizan para su cerramiento 
materiales flexibles, no permanentes, de forma que admiten soportes estructurales más ligeros. La expansión de este tipo de invernaderos se potencia con la crisis energética y con la concienciación social de la limitación de los recursos naturales. Se abre aquí una nueva línea de evolución de invernaderos que presentan, respecto a los de material de cerramiento rígido, mayores ventajas técnicas y económicas, derivadas de la flexibilidad de los cerramientos y de su menor peso, permitiendo unas estructuras más económicas.

La tercera vía de evolución en la tecnología de invernaderos la constituyen unas estructuras de bajo coste de inversión, realizadas artesanalmente con materiales poco elaborados. Estos invernaderos se caracterizan por mejorar su microclima de forma pasiva, actuando como captadores solares que consiguen aumentar la integral térmica en su interior.

\section{En el XII Congreso Internacional de Plásticos en} Agricultura, celebrado en Granada en 1992, se pone de manifiesto el auge en el consumo mundial de plásticos para invernadero $y$, por tanto, la masiva extensión de las estructuras ligeras (vías $2^{\mathrm{a}}$ y $3^{\mathrm{a}}$ de la evolución antes descrita). En España la superficie protegida superaba las $45.000 \mathrm{Ha}$.

TABLA I

\begin{tabular}{|c|c|}
\hline Grandes zonas & Ha. \\
\hline Área mediterránea & 105.300 \\
\hline Resto de Europa & 16.700 \\
\hline Continente americano & 13.000 \\
\hline Área oriental & 138.000 \\
\hline Total & 273.000 \\
\hline
\end{tabular}

Superficie (Ha) ocupadaen el mundo porinvernaderos plásticos. (Castilla, 1994).

En Almería, el aumento de la superficie protegida y sus necesidades de agua provocó la sobreexplotación de los acuíferos, por lo que en 1983 las autoridades españolas prohibieron el aumento de la superficie regada en el Campo de Dalías, que cuenta con más de 15.000 Ha de invernadero, situación actualmente vigente. No obstante, la situación real indica que su construcción ha seguido aumentado en los últimos años.

La importancia del sector quedaría reflejada en el siguiente dato: en 1992, las casi $23.000 \mathrm{Ha}$ de invernadero existentes en Almería han producido cerca de un billón de US\$. (López Gálvez, 1994)

A partir del 1 de enero de 1993 se eliminaron las restricciones impuestas por la CEE a las frutas y hortalizas españolas; esta nueva situación junto a los avances tecnológicos, sobre todo en ahorro de agua de riego: el gasto de agua ha pasado de más de 7.000 $\mathrm{m}^{3} / \mathrm{Ha}$ año en 1982 a $5.500 \mathrm{~m}^{3} / \mathrm{Ha}$ año según datos actuales (López-Gálvez et al., 1994); y pese a la prohibición de nueva construcción, que no afecta al Levante almeriense, ha permitido que el sector de la construcción de invernaderos en Almería, compuesto básicamente por 18 empresas, facturase, en 1994 , alrededor de los 15.000 millones de pesetas.

Se prevé que esta cifra aumente en los próximos años, pues si bien se estima que, para mantener la productividad de los invernaderos actuales, se precisará una cantidad global de agua de $140 \mathrm{Hm}^{3}$, cantidad que, actualmente no está garantizada, las esperanzas se focalizan hacia el Levante almeriense, que podrá regarse muy pronto. La presa al norte del Campo de Níjar ya está construida, cerrada y llena, faltando sólo la red de distribución.

Por otra parte, los agricultores de la zona están tomando consciencia de que sus invernaderos tradicionales no son competitivos en la actualidad y están comenzando a sustituirlos por otros más funcionales.

Este entorno socioeconómico y político, junto con las expectativas de desarrollo del sector antes comentadas, justifican la necesidad de afrontar estudios para incorporar estructuras novedosas que, por un lado reduzcan los costes de producción, que presenten un mejor comportamiento estructural, y que mejoren las condiciones de captación de la energía. Es dentro de estos objetivos donde se enmarca la presente tesis doctoral.

La falta de resistencia al viento es la causa de numerosos fallos estructurales en los invernaderos convencionales, provocando situaciones económicas críticas en el sector. Esto, unido a la crisis por la que atraviesa en estos momentos el sector agrario, aparte de otro tipo de medidas, hace especialmente importante la labor de la Investigación como fuente de innovación para mejorar la rentabilidad del sector en su doble vertiente, diversificación y mejora de la calidad en la producción por un lado y reducción de costes por otro, tanto los de producción como los de inversión en estructura.

A pesar de la evolución empírica de estos invernaderos, al no haber sido diseñado originariamente para su función actual, plantea problemas estructurales y agronómicos entre los que caben destacar (Pérez et al., 1992):

- Falta de seguridad ante el viento, con levantamiento de las cimentaciones a tracción (muertos y 
amagados) y hundimiento de los que trabajan a compresión (pies derechos), destensando la estructura, lo que obliga a un retensado periódico. También se producen frecuentes averías y, en más ocasiones de las deseadas, la destrucción de la instalación.

- Exceso de soportes (rollizos y amagados) que reducen la superficie útil, dificultan la previsible necesidad de mecanización de los cultivos e imposibilita una instalación sencilla de doble techo, cuyas ventajas han quedado patentes en diversos estudios (Chandra \& Albright, 1980) para disminuir los riesgos de inversión térmica.

- La baja altura de la instalación evita la formación de un gradiente térmico suficiente para una buena ventilación cenital, provocando excesos de humedad y la consecuente aparición de enfermedades criptogámicas.

Otro problema relacionado con la estructura que debe abordarse es la mejora de las condiciones radiativas que va a redundar en una mayor productividad, sobre todo en invernaderos de clima pasivo. La mejora de la transmisividad de la radiación solar se puede conseguir mediante materiales de cerramiento que mejoren esta propiedad y/o adecuando la orientación y forma de la cubierta a la situación geográfica de cada lugar (LópezGálvez 1994).

\section{OBJETIVO}

El objetivo general es el estudio teórico y experimental de un prototipo de invernadero basado en una estructura resistente plana, ligera, elástica y altamente deformable. $\mathrm{Su}$ diseño persigue resolver las limitaciones de los invernaderos tradicionales y proponer dicha estructura, previa comprobación de su viabilidad técnica y económica, como una solución alternativa de invernadero de estructura económica que suponga una innovación tecnológica.

Para la consecución de este objetivo, se han planteado una serie de objetivos específicos que permiten ir completando las distintas fases del trabajo.

\section{- Diseño de la estructura básica bajo criterios de} resistencia, funcionalidad y economía.

- Generación de un modelo físico para el cálculo teórico de los distintos estados de tensiones y deformaciones, frente a solicitaciones internas, durante las distintas fases del montaje, así como, posteriormente, frente a estados definidos de cargas exteriores.
- Diseño, desarrollo y construcción de un prototipo experimental.

- Diseño y desarrollo de un sistema electrónico de medida y almacenamiento de datos de los estados tensionales de la estructura frente a temperatura, velocidad y dirección del viento.

\section{DISEÑO METODOLÓGICO}

\subsection{Introducción}

En este capítulo se van a plantear las bases para el diseño de la nueva estructura de cubierta; partiendo de una idea básica, que se sustenta en la teoría de hilos, llegamos a establecer los criterios geométricos de la nueva estructura en base a los aspectos formal y estructural.

Seguidamente se aborda su estudio elástico mediante el planteamiento de un modelo físico que, de forma simplificada, consiste en la sustitución de cada tramo de cable por un resorte de constante elástica (S.E)/1. La resolución matemática de dicho modelo, mediante un proceso iterativo semejante al método de Hardy-Cross, permite conocer las tensiones que soportan los cables en las distintas fases del montaje, así como su comportamiento estructural frente a diferentes hipótesis de carga, principalmente acciones de viento.

\subsection{Bases para el diseño de la nueva estructura}

Las cubiertas flexibles de los invernaderos tradicionales "tipo parral" se conforman en base a una malla plana de hilos pretensados.

Los años de experiencia demuestran que esta cubierta no admite vanos diáfanos por encima de los $8 \mathrm{~m}$ y que, incluso así, todavía existen demasiados casos donde esta estructura no aguanta los efectos de viento (cargas perpendiculares al plano de cubierta).

Este problema surge como consecuencia de que un hilo flexible, para resistir un esfuerzo perpendicular, se ha de deformar y alcanzar una alta tensión de manera que la componente perpendicular de ésta iguale al esfuerzo exterior. Distinto sería si la disposición inicial del hilo no fuera horizontal, sino que presentara una componente en la dirección de la carga exterior. Ahora bien, el problema se plantea a la hora de poder desarrollar una forma estructural que permita adoptar la disposición de hilo previamente deformado.

Una posibilidad es la que se puede observar en la Fig.1. Aunque la estructura tiene la apariencia de una "cercha" típica, funciona de una forma muy distinta. 


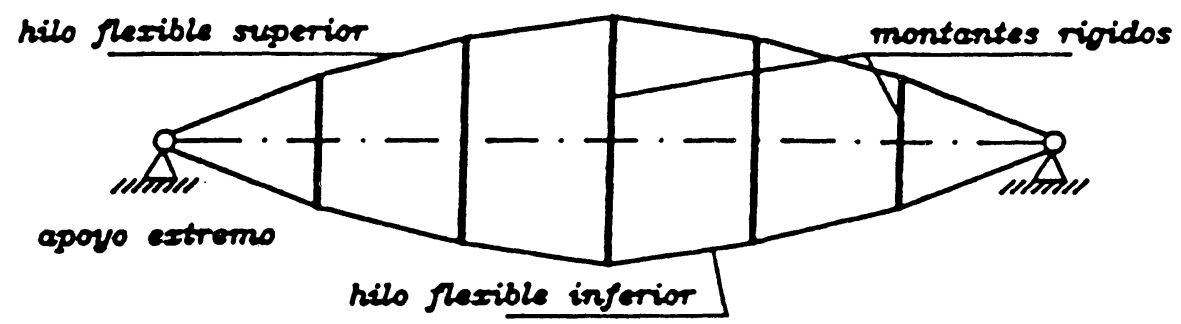

Fig. 1

Para mantener la forma, ambos hilos han de estar traccionados, contrarrestándose las componentes verticales de estas tracciones con las compresiones a que se ven sometidos los montantes rígidos.

Así, las cargas verticales descendentes exteriores (gravitatorias) provocarán una disminución de la tensión del hilo superior y un aumento en la del hilo inferior. De igual forma, las cargas verticales ascendentes (de viento) provocarán el tensado del hilo superior y el destensado del hilo inferior (Figura 2).
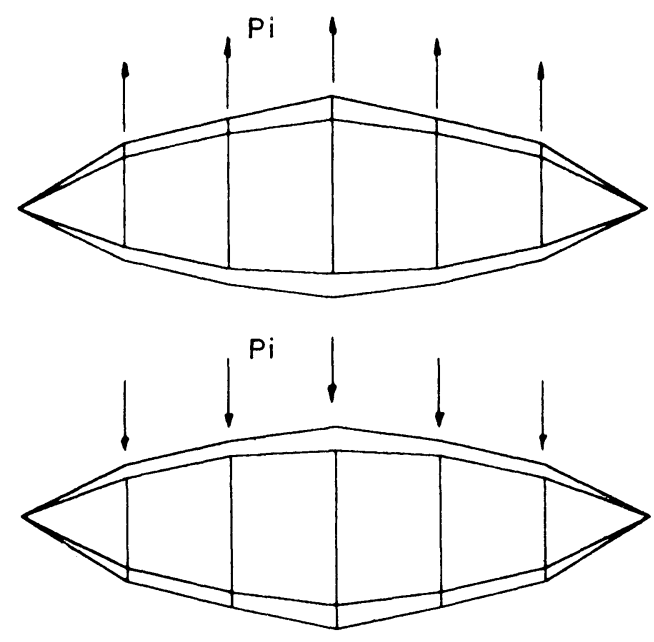

Fig. 2

Esta estructura plana pretensada tiene una capacidad mucho mayor de resistir esfuerzos exteriores en su plano que un simple hilo horizontal. Su dimensionamiento ha de ser tal, que en la zona de tracciones no se agote la tensión de rotura minorada por el coeficiente de seguridad aplicado y que paralelamente, en la zona de compresiones, la tracción de pretensado no disminuya excesivamente para que no aparezcan deformaciones en la estructura.

Ahora bien, así como la estructura puede resistir fuertes cargas en su plano, no ocurre igual en el plano perpendicular a ella. Es decir, se necesitan unos elementos de arriostramiento que mantengan la forma y que resistan las solicitaciones externas perpendiculares al plano de la estructura. Esto se puede resolver con la fijación de los nudos superiores de los montantes rígidos a hilos perpendiculares que trabajen a modo de "correas".

Como se puede observar, el modelo estructural que aparece es análogo al de una nave tradicional. Es decir, se pretende que las correas sean elementos de arriostramiento transmitiendo las cargas verticales a la cercha plana que se considera como elemento principal de resistencia.

Aceptar este esquema estructural implica basar el estudio en el comportamiento plano de la cercha. A tal fin se ha de realizar un modelo matemático para calcular la respuesta elástica ante un sistema de cargas definido. Es éste el sistema de cálculo realizado que se describe en apartados posteriores.

No obstante, cabe indicar que la "estructura flotante" que resulta si se consideran cerchas y correas como elementos principales de resistencia, responde a dos mallas en el espacio. Una, la inferior, sería la formada por todos los hilos inferiores de las cerchas, y la otra, la superior, que estaría constituida por los hilos superiores de las cerchas más las correas; es decir, sería una malla formada por dos entramados de "nervios" perpendiculares formando rectángulos de las dimensiones que arrojen la distancia entre montantes y separación entre cerchas

Considerar este segundo esquema estructural resulta mucho más complejo a la hora de establecer el modelo matemático de cálculo. Por otro lado, considerando el primer esquema estructural se está del lado de la seguridad, pues, en definitiva, no se contempla la contribución de las correas a la resistencia de la estructura frente a cargas verticales.

\subsection{Geometría de la estructura}

Las dimensiones de los montantes definirán la forma de la cercha, teniendo en cuenta que ésta se verá, además, afectada por la relación de las tensiones de los cables superior e inferior, pues dicha relación influirá en el porcentaje de la cercha que queda por encima de la horizontal. 
La forma que se pretende dar a la cercha persigue varios objetivos:

- Aspecto formal: la cercha ha de soportar la cubierta y ésta ha de ser cóncava para poder evacuar las aguas de lluvia.

- Aspecto estructural: se pretende que el cable superior quede en su montaje con una forma semejante a las deformaciones que, teóricamente, produce una carga uniformemente repartida sobre un hilo flexible, formando en sus extremos un ángulo suficiente, con la horizontal, que proporcione unas componentes verticales de la tensión capaces de absorber dichas cargas.

Por otra parte, una superficie de cubierta curva es más eficiente, en la captación de la radiación solar a lo largo del año, que una superficie captadora plana, en condiciones estáticas.

Es por lo descrito anteriormente por lo que se decide que la geometría de la cercha adopte una forma curva, obviamente formada por tramos rectos. Falta por decidir los valores de los referidos ángulos con la horizontal, así como las dimensiones de los montantes rígidos que determinarán la curvatura.

El ángulo de deformación previa que ha de formar el hilo en sus extremos, de las dimensiones y de las cargas a las que vaya ha estar sometido, calculado para los siguientes datos, es de $\alpha=25^{\circ}$ :

- Longitud del vano: $\mathbf{L}=16 \mathrm{~m}$

- Separación entre cerchas: $\mathbf{a}=3 \mathrm{~m}$.

- Tensión máxima admisible en el cable: $\mathbf{t}=3.000 \mathrm{kp}$.

- Presión dinámica del viento: $=47^{\prime} 5 \mathrm{kp} / \mathrm{m}^{2}$

Para la curvatura de la cubierta (Fig. 3), vamos a calcular el montante central suponiendo que la sección transversal se aproxima a un arco de circunferencia ${ }^{(*)}$.

Estimando que la flecha máxima del cable inferior puede ser alrededor de $0,5 \mathrm{~m}$, asignamos al montante central el valor total de $2,27 \mathrm{~cm}$ (Fig. 4).

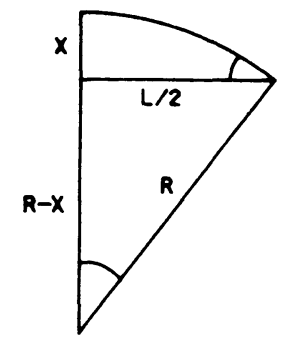

Fig. 3

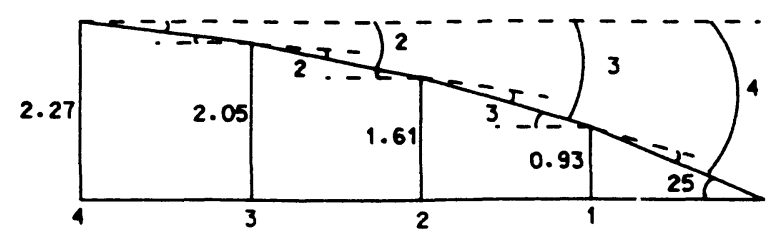

Fig. 4

Para el cálculo de los otros montantes debemos desechar la circunferencia, pues ésta supondría quedarnos sin pendiente en los tramos próximos a la cumbrera, con el peligro de formación de bolsas de agua. Así, pues, tomamos otro criterio de diseño que supone iguales variaciones de pendiente en todos los tramos. De esta forma, queda algo parecido a una circunferencia, con una pequeña pendiente en los tramos de cumbrera y mayores pendientes conforme nos alejamos de la misma, que son los tramos que más agua han de evacuar.

En cuanto al número de montantes, se establece en 7 , de forma que quedan 8 tramos $\mathrm{y}$, por tanto, un vano máximo del cable pórtico de $2 \mathrm{~m}$.

Según dicho criterio y el número de montantes establecido, las dimensiones de éstos serán:

$$
4 \alpha=25^{\circ} ; \alpha=6,25
$$

Montante 1: $\mathrm{L}_{1}=2 \operatorname{tg} 25^{\circ}=0,93 \mathrm{~m}$

Montante $2: \mathrm{L}_{2}=\mathrm{L}_{1}+2 \operatorname{tg}(3 \alpha)=1,61 \mathrm{~m}$

Montante 3: $\mathrm{L}_{3}=\mathrm{L}_{2}+2 \operatorname{tg}(2 \alpha)=2,05 \mathrm{~m}$

Montante 4: $\mathrm{L}_{4}=\mathrm{L}_{3}+2 \operatorname{tg} \alpha=2,27 \mathrm{~m}$

Por tanto, se prevé que la geometría de la cercha podría quedar de la manera que presenta la Figura 5.

$(*)$

$$
\begin{aligned}
& \mathrm{R}-\mathrm{x}=\sqrt{\mathrm{R}^{2}-\left(\frac{\mathrm{L}}{2}\right)^{2}} ; \quad \frac{\mathrm{L}}{2}=\mathrm{R} \operatorname{sen} \alpha \quad ; \quad \mathrm{R}=\frac{\mathrm{L}}{2 \operatorname{sen} \alpha} \\
& \mathrm{x}=\mathrm{R}-\sqrt{\mathrm{R}^{2}-\mathrm{R}^{2} \operatorname{sen}^{2} \alpha} ; \quad \mathrm{x}=\mathrm{R}(1-\cos \alpha) \mathrm{y} \text { sustituyendo } \mathrm{R} \text { por su valor : } \\
& \mathrm{x}=\frac{\mathrm{L}}{2} \frac{1-\cos \alpha}{\operatorname{sen} \alpha} \quad \text { para } \mathbf{L}=16 \mathrm{~m} \text { y } \alpha=25^{\circ}, \mathrm{x}=177 \mathrm{~m} .
\end{aligned}
$$




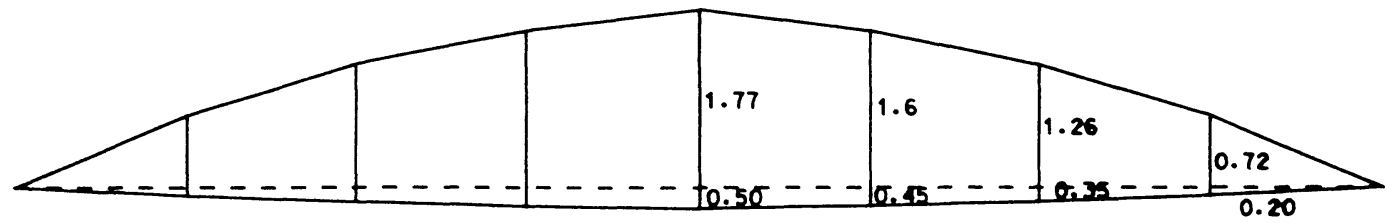

Fig. 5

Se ha considerado que todos los montantes tienen el mismo porcentaje sobre la horizontal que el montante central, manteniéndose, así, el criterio de variación de pendiente constante en los tramos inferiores.

No obstante, la forma, en cuanto al porcentaje de los montantes que quedan por encima de la horizontal, dependerá, como ya se comentó antes, de la relación entre las tensiones de montaje de los cables superior e inferior y, por supuesto, de las cargas a las que se someta la estructura.

\subsection{Estudio elástico de la estructura. Modelo físico}

\subsubsection{Modelo fisico empleado}

Para el análisis de la estructura se han considerado como invariantes las longitudes naturales de los cables comprendidos entre nudos consecutivos (para una misma temperatura).

Dichas longitudes se pueden determinar a partir de las fases constructivas de la estructura. De este modo, teniendo en cuenta la ley de Hooke, para cada tramo de cable:

$$
F=\left(\frac{S E}{1}\right) \Delta 1
$$

resultando que la fuerza que cada cable realiza es directamente proporcional a su deformación. Así, el modelo físico consiste en la sustitución de cada tramo de cable por un resorte de constante elástica (S.E)/I.

Ahora bien, el comportamiento de un cable puede asimilarse al de un resorte sólo bajo condiciones de tracción, pues en compresión, el cable está destensado mientras el resorte presenta una situación simétrica a la tracción. Así, pues, asignaremos valor cero a la fuerza de aquel tramo cuya longitud sea inferior a la longitud natural, siendo ésta la del supuesto resorte en equilibrio. De esta forma, la determinación de la posición de equilibrio y los estados tensionales cuando la estructura está sometida a unos esfuerzos $\mathbf{F}_{\mathbf{p}}$, se realiza resolviendo el problema de estática que se representa en la Figura 6.

Siendo $\mathbf{K}_{1}, \ldots, \mathbf{K}_{2 n+2}$ las constantes elásticas asociadas a cada tramo de cable; $l_{1}, \ldots, l_{n}$ las longitudes de cada uno de los montantes.

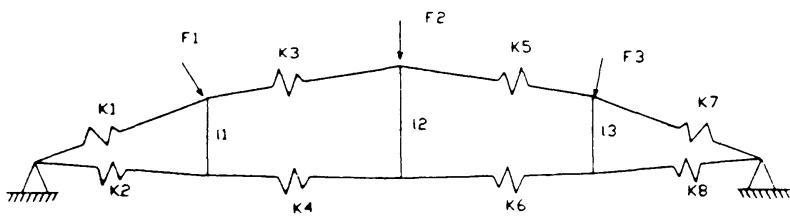

Fig. 6

El sistema posee $3 n$ grados de libertad (siendo $\mathrm{n}$ el número de montantes). Lo que implica un elevado número de ecuaciones ya en los casos más sencillos en que el número de montantes es pequeño.

Es por esto que para la resolución se ha escogido un sistema iterativo basado en la posición de equilibrio de un montante i supuestas fijas y conocidas las coordenadas de las barras adyacentes $\mathbf{i}-\mathbf{1}, \mathbf{i}+\mathbf{1}$, lo que es equivalente al problema representado en la Figura 7. En este caso, sólo se tienen 3 grados de libertad.

La resolución de este subproblema se ha abordado minimizando la función de potencial energético asociado a las fuerzas que actúan sobre dicha barra.

Exponemos el planteamiento de este problema sobre la nomenclatura simplificada que se especifica en la Figura 8 de cara a hacerlo más fácilmente comprensible.

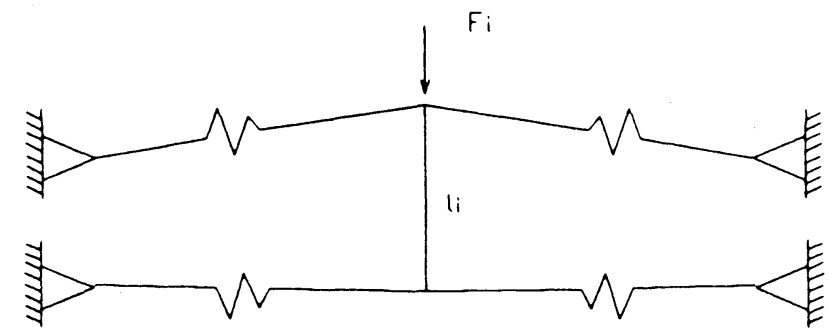

Fig. 7

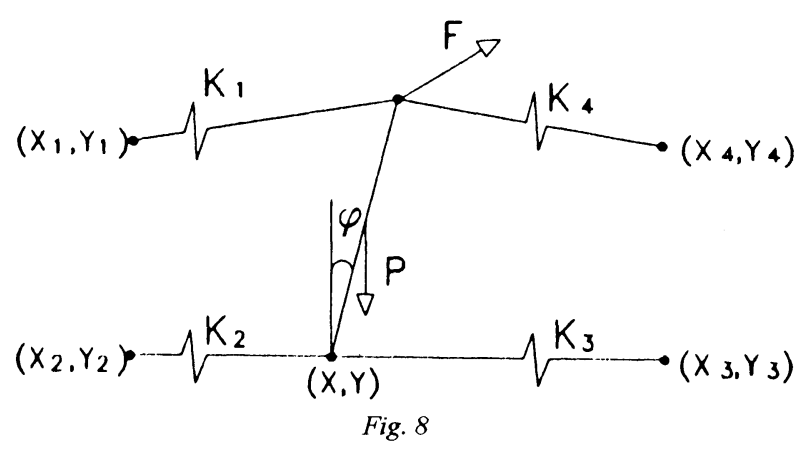


Las coordenadas libres escogidas han sido $\mathrm{x}, \mathrm{y}, \mathrm{n}$. La función potencial queda constituida por cuatro términos elásticos, uno gravitatorio y el asociado a la fuerza exterior $\mathrm{F}$, siendo las funciones potenciales elásticas:

$$
v_{E 1}=\frac{1}{2} k_{1}\left[\sqrt{\left(x_{1}-x-d \operatorname{sen} \varphi\right)^{2}+\left(y_{1}-y-d \cos \varphi\right)^{2}}-l_{1}\right]^{2}
$$

$$
v_{E 2}=\frac{1}{2} k_{2}\left[\sqrt{\left(x_{2}-x\right)^{2}+\left(y_{2}-y\right)^{2}}-l_{2}\right]^{-}
$$

$$
v_{E 3}=\frac{1}{2} k_{3}\left[\sqrt{\left(x_{3}-x\right)^{2}+\left(y_{3}-y\right)^{2}}-l_{3}\right]^{-}
$$

$v_{E 4}=\frac{1}{2} k_{4}\left[\sqrt{\left(x_{4}-x-d \operatorname{sen} \varphi\right)^{2}+\left(y_{4}-y-d \cos \varphi\right)^{2}}-l_{4}\right]^{2}$

\section{El potencial gravitatorio viene expresado por}

$$
v_{G}=P\left(y+\frac{1}{2} d \cdot \cos \varphi\right)
$$

El potencial asociado a la fuerza exterior $F\left(F_{x}, F_{y}\right)$ viene expresado por

$$
\nu_{F}=-(x+d \operatorname{sen} \varphi) F_{x}-(y+d \cos \varphi) F_{y}
$$

y por tanto: $\mathrm{v}(\mathrm{x}, \mathrm{y}, \mathrm{n})=\mathrm{v}_{\mathrm{E} 1}+\mathrm{v}_{\mathrm{E} 2}+\mathrm{v}_{\mathrm{E} 3}+\mathrm{v}_{\mathrm{E} A}+\mathrm{v}_{\mathrm{G}}+\mathrm{v}_{\mathrm{F}}$

La resolución del sistema

$$
\frac{\partial v}{\partial x}=0: \frac{\partial v}{\partial y}=0 ; \frac{\partial v}{\partial z}=0
$$

no resulta abordable analíticamente por lo que se ha acudido al método numérico de Newton, en que partiendo de una solución $\mathrm{x}^{\circ}, \mathrm{y}^{0}, \varphi^{\circ}$ se generase las series convergentes $\mathrm{x}^{\mathrm{n}}, \mathrm{y}^{\mathrm{n}}, \mathrm{n}^{\mathrm{n}}$ mediante

$$
\left(\begin{array}{c}
x^{n+1} \\
y^{n+1} \\
\varphi^{n+1}
\end{array}\right)=\left(\begin{array}{c}
x^{n} \\
y^{n} \\
\varphi^{n}
\end{array}\right)-\left[\begin{array}{lll}
\frac{\partial^{2} v}{\partial x^{2}} & \frac{\partial^{2} v}{\partial x \partial y} & \frac{\partial^{2} v}{\partial x \partial \varphi} \\
\frac{\partial^{2} v}{\partial x \partial y} & \frac{\partial^{2} v}{\partial y^{2}} & \frac{\partial^{2} v}{\partial y \partial \varphi} \\
\frac{\partial^{2} v}{\partial x \partial y} & \frac{\partial^{2} v}{\partial y \partial \varphi} & \frac{\partial^{2} v}{\partial \varphi^{2}}
\end{array}\right]_{\left(\begin{array}{l}
x=x^{n} \\
y=y^{n} \\
\varphi=\varphi^{n}
\end{array}\right)}\left(\begin{array}{c}
\frac{\partial v}{\partial x} \\
\frac{\partial v}{\partial y} \\
\frac{\partial v}{(1} \\
y=y^{n} \\
\varphi=\varphi^{n}
\end{array}\right)
$$

Así, la resolución del problema representado en la Figura 7 se basa en la asignación de unas coordenadas iniciales a los nudos de la estructura. Sucesivamente dichas coordenadas se van modificando. El método de modificación consiste en el recálculo de las coordenadas de cada barra, según se esquematizó en la Figura 8. Esto es, en cada iteración se calculan las coordenadas de cada barra, para su nueva posición de equilibrio, supuestas fijas las posiciones de las demás barras. Estas nuevas coordenadas se asignan a la barra y se fija, liberándose la siguiente. Las nuevas coordenadas de la barra anterior habrán modificado el equilibrio $\mathrm{y}$, por tanto, las coordenadas de la siguiente, que se calculan nuevamente entrando en un proceso iterativo semejante al método de Hardy-Cross.

Este proceso iterativo es posible ya que la posición buscada es de equilibrio estable o de potencial mínimo, por lo que las soluciones convergen a esos valores. Las iteraciones terminan cuando el error máximo en la convergencia es inferior a $0,001 \mathrm{~mm}$.

\subsubsection{Resolución del modelo fisico}

Para automatizar la resolución del modelo físico de la estructura resistente de cubierta hemos desarrollado un programa de cálculo que hemos denominado PRETENSA, que tiene la peculiaridad de seguir los mismos pasos que el proceso constructivo, determinando los estados tensionales de los cables y sus estiramientos en cada fase.

\section{Primera fase (Posición inicial, Figura 9):}

En esta fase sólo interviene el hilo inferior sometido a la tensión inicial de proyecto y al que se le han colgado las traviesas equidistantes.

Con los datos que afectan al hilo inferior y a las traviesas se determinan las longitudes naturales de los tramos entre traviesas de dicho hilo, así como el estado de tensiones y deformaciones en esta primera fase constructiva de la estructura. 
En esta fase el programa ofrece la posibilidad de generar un fichero de dibujo; en caso afirmativo lo archivará como fichero gráfico *.DXF.

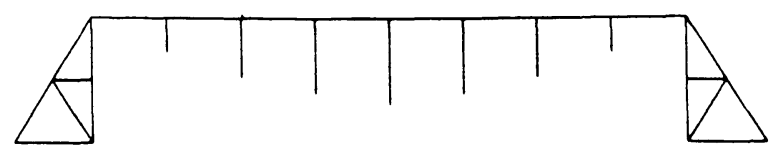

Fig. 9

Segunda fase (Posición invertida, Figura 10):

Consiste en el tendido del hilo superior y su tensado al valor constructivo, manteniendo las traviesas verticales. Se habrá obtenido la estructura en posición invertida.

Tras la determinación de las longitudes naturales del hilo superior y los estados de tensión y deformación de la estructura en esta posición, el programa ofrece la posibilidad de generar, además del fichero gráfico, un fichero de reporte.

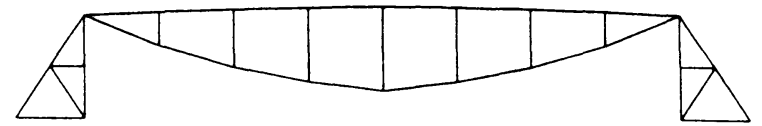

Fig. 10

Tercera fase (Posición normal, Figura 11):

Tras haber girado la estructura hasta su posición definitiva, se determinan las coordenadas de los nudos y los estados tensionales y de deformación para la nueva posición de equilibrio. Igualmente, se ofrece la posibilidad de generar un fichero de dibujo y otro de reporte que recogerá las características de la estructura en posición normal.

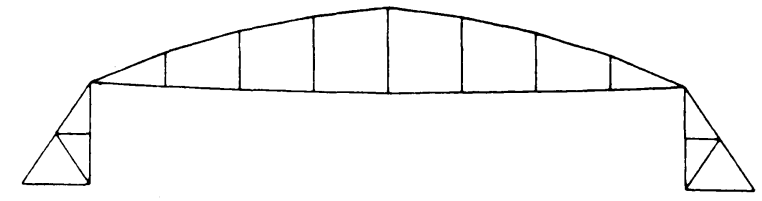

Fig. 11
Todos los cálculos anteriores se realizan para una temperatura de $20^{\circ} \mathrm{C}$, que es la temperatura media estimada durante el montaje. No obstante el programa nos ofrece la posibilidad de calcular la cercha en posición normal para otros valores de la temperatura.

En caso afirmativo y tras introducir el valor deseado de la temperatura en grados centígrados, el programa efectuará la modificación de las longitudes naturales, por dilatación térmica, de acuerdo con la expresión:

$\mathrm{L}=\mathrm{L}_{0}[1+\alpha(\mathrm{T}-20)]$ donde $\alpha$ es el coeficiente de dilatación térmica.

Tras recalcular la estructura y ofrecernos los ficheros de salidas, nos permite nuevamente modificar la temperatura. En caso negativo, el programa salta a la siguiente fase.

\section{Cuarta fase (Figura 12):}

En este punto el programa está dispuesto para determinar los estados tensionales y de deformación de la estructura bajo cualquier hipótesis de esfuerzos sobre los nudos superiores. Si se quiere realizar dicho análisis, bastará con introducir las componentes horizontales y verticales del esfuerzo en cada nudo.

Estos cálculos los realiza nuevamente a la temperatura de $20^{\circ} \mathrm{C}$ y, tras ofrecernos el fichero gráfico y de reporte, nos permite recalcular para otros valores de T. En caso afirmativo y tras el análisis, podemos modificar el valor de $\mathbf{T} \mathbf{y}$, en caso negativo, podremos abordar nuevas hipótesis de carga. Saldremos del programa negándonos a esto último.

En cada fase el programa nos informa a qué temperatura está analizando la estructura y un porcentaje creciente indica los progresos en el proceso de iteración. Al final de cada análisis podemos visualizar en pantalla las tensiones medias en ambos cables.

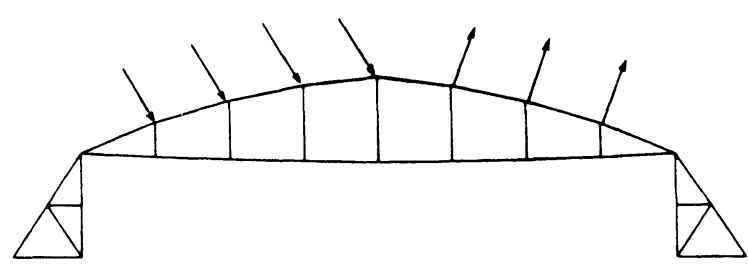

Fig. 12 


\section{ESTUDIO EXPERIMENTAL}

Para el estudio experimental se ha construido un prototipo de invernadero financiado por Resolución de Ayuda del Instituto de Fomento de Andalucía aprobada con fecha de 23 de diciembre de 1992, al amparo del Programa de Interés Comunitario de Almería-Levante.

En este apartado se realiza el proyecto del prototipo, con el fin de dar una solución técnica a todos los problemas que se pueden plantear para la materialización como invernadero de una estructura totalmente novedosa. A lo largo del mismo se va a realizar el dimensionado definitivo de los distintos elementos, a fin de adaptarlos a las características tanto geométricas de la parcela, como geotécnicas en cuanto al diseño de la cimentación y de los postes definitivos. La construcción y puesta a punto de una estructura de esta índole requerirá, asimismo, del diseño de una serie de elementos que en un estudio global podrían considerarse como accesorios o de importancia menor, pero que de hecho, de su buen diseño depende en gran medida la viabilidad técnica del producto final: buen ejemplo de éstos serían las uniones de cerchas y pilares, las propias uniones de montantes y cables dentro de la estructura, la forma y disposición de los elementos de desagüe, etc.

El prototipo construido está formado por tres naves adosadas, con una luz de $14,4 \mathrm{~m}$ cada una, asimismo se ha dispuesto una banda perimetral de $2 \mathrm{~m}$, con lo que resulta una anchura total de 47,2 $\mathrm{m}$. La longitud total de las naves es múltiplo de 3,25 m (separación entre cerchas), más la banda perimetral, obteniéndose una longitud de $39,75 \mathrm{~m}$.

Con el fin de contrastar el modelo físico de funcionamiento de la estructura por un lado y, por otro, la bondad de las hipótesis de carga propuestas en el diseño (coeficientes de carga, etc), es necesario conocer los estados tensionales de la estructura de cubierta durante la fase de montaje y la fase de funcionamiento, ante condiciones variables de temperatura y de carga (velocidad y dirección de viento).

Se ha considerado necesario medir las tensiones en los cables superior e inferior de, al menos, dos cerchas significativas y la temperatura en cuatro puntos, a diferentes alturas, dentro del invernadero. Asimismo se han de conocer las componentes del vector viento.

Los datos experimentales obtenidos por el sistema automático de adquisición de datos pueden visualizarse, a modo de ejemplo, en la Figura 13

Puede apreciarse el comportamiento lógico, a nivel cualitativo, en cuanto a la influencia de las variaciones de temperatura sobre las tensiones, en el sentido de aumentar éstas cuando disminuye aquélla y viceversa; y el tensado del cable superior, simultáneo al destensado del inferior de la cercha correspondiente, como respuesta a las acciones del viento. Aunque, como podemos observar en la Figura 13, existe un movimiento vibratorio que hace alternar la situación antes descrita, es decir, se simultanean el tensado del superior frente al destensado del inferior y viceversa.

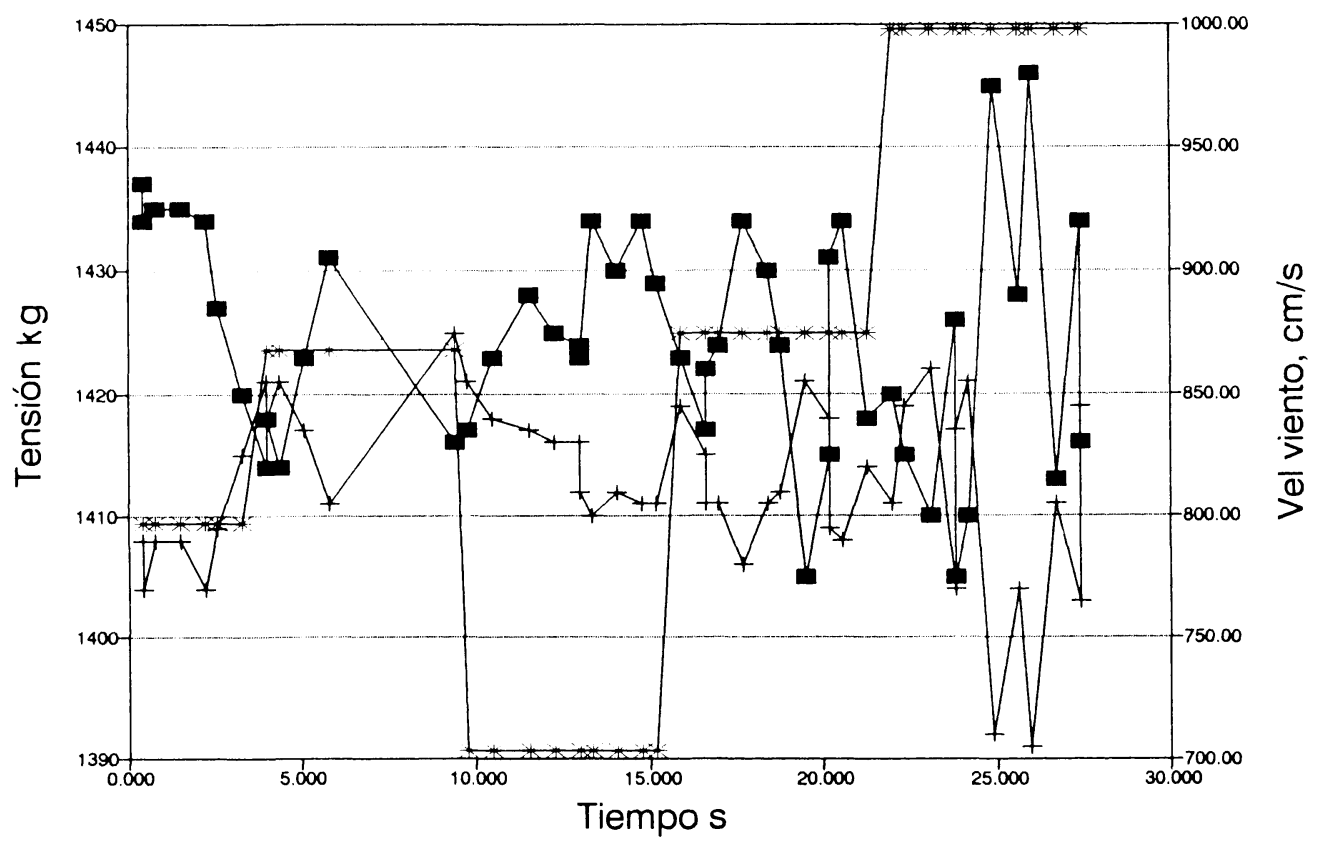

$\rightarrow$ T. Inferior $\quad+$ T. Sup. $+1350 \mathrm{~kg} \rightarrow$ Vel. viento 


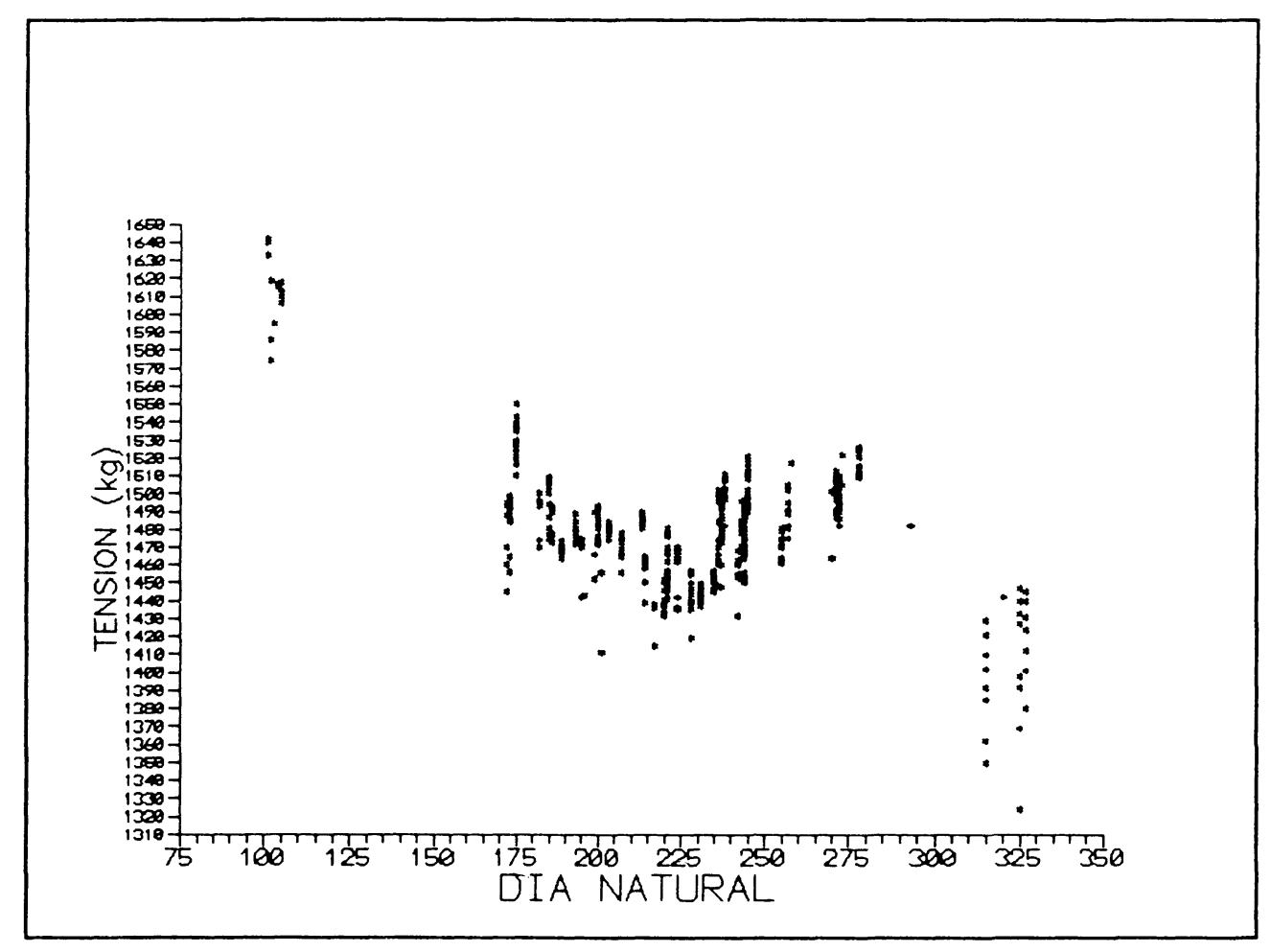

Fig. 14

Para evitar la influencia de las variaciones de temperatura y de la velocidad del viento sobre las tensiones, se han filtrado los ficheros representando los valores de las tensiones de registros correspondientes a intervalos definidos de temperatura y velocidad del viento, mostrándose a modo de ejemplo en la Figura 14.

En dicha figura se observa una oscilación de los valores de las tensiones, debido a que para cada día se representan todos los valores de tensiones obtenidos en un intervalo de temperatura y velocidad de viento.

\section{SIMULACIÓN DE LA ESTRUCTURA CON EL MODELO FÍSICO}

En la Figura 15 se muestra, a modo de ejemplo, el comportamiento de la estructura, según los valores teóricos calculados con el modelo físico, para las mismas condiciones que los datos experimentales representados. Puede observarse que las diferencias, entre los valores teóricos y experimentales, son porcentualmente mayores en las tensiones del cable superior. Estas diferencias son debidas a la carga que supone el conjunto de correas, mallas y plástico (Fig. 16), que ayuda al cable superior en su trabajo y que no han sido tenidas en cuenta en los cálculos, quedando del lado de la seguridad. Dado el comportamiento elástico del conjunto formado por las correas, mallas y plástico, los esfuerzos que éstas realizan deben ser proporcionales a las deformaciones. Ello explica que las mencionadas diferencias, entre valores teóricos y experimentales, se incrementen con valores crecientes de la velocidad del viento.

Estas representaciones gráficas muestran la imposibilidad de estimar la probabilidad de que se alcancen los valores teóricos. No obstante. su aspecto indica que las probabilidades de fallo, para los coeficientes eólicos considerados, son muy pequeñas.

\section{INNOVACIÓN TECNOLÓGICA DEL INVERNADERO}

El diseño de su estructura le confiere indudables ventajas y nuevas posibilidades frente a estructuras convencionales:

\section{Gran resistencia:}

- Frente a acciones de viento.

\section{Diafanidad:}

- Posibilidad de despliegue automatizado de doble techo, en una sola pieza por nave, que le confiere mayor hermeticidad (Fig. 17).

- Operatividad de maquinaria y nuevas posibilidades de mecanización.

\section{Altura:}

- Posibilidad de proyectar instalaciones de mayor altura, 


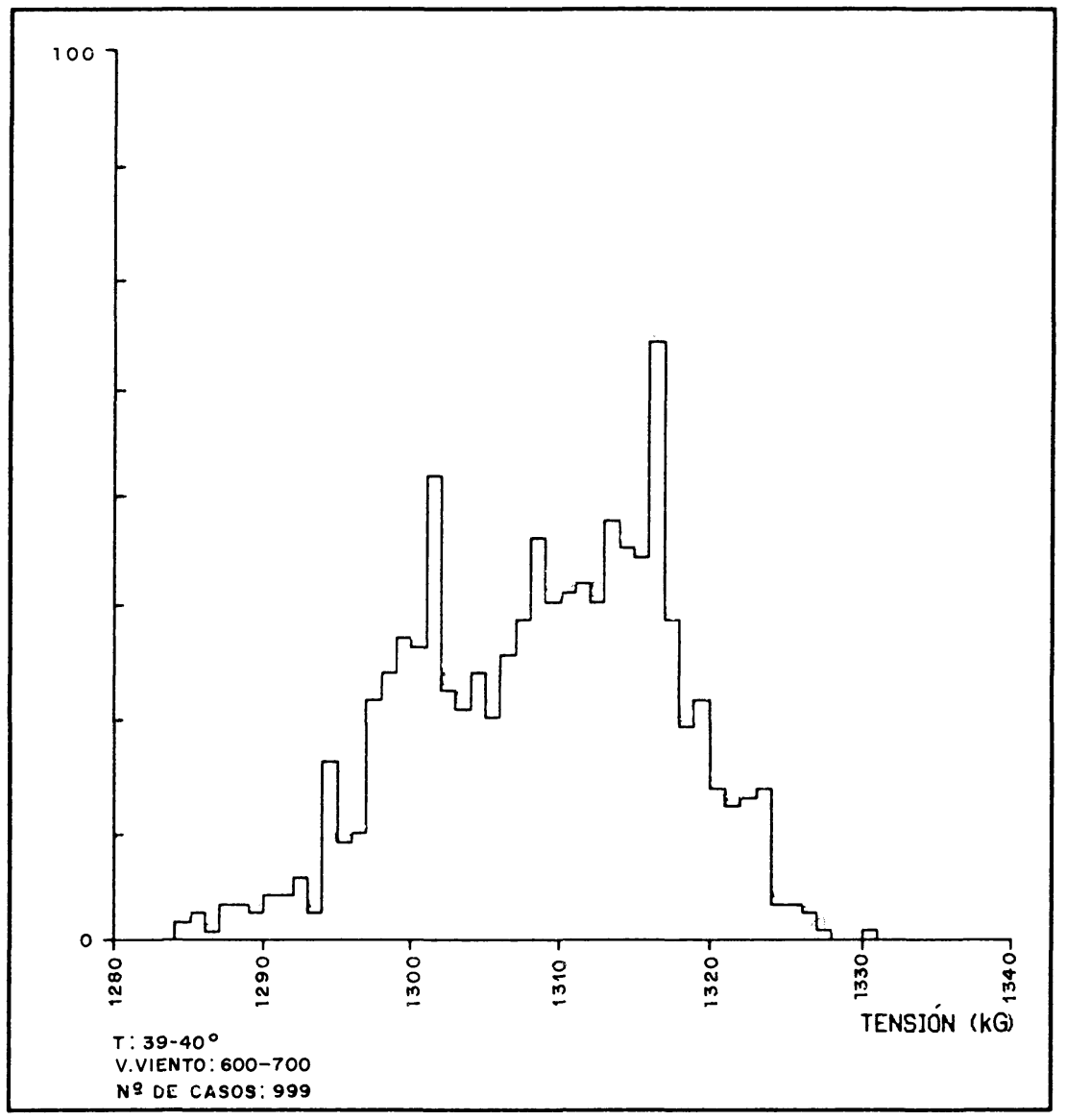

Histograma de Tensiones. Cercha-1 cable inferior. $\mathrm{T}=39-40^{\circ}, \mathrm{VV}=6-7 \mathrm{~m} / \mathrm{s}$. VALOR TEÓRICO: $1.387 \mathrm{~kg}$.

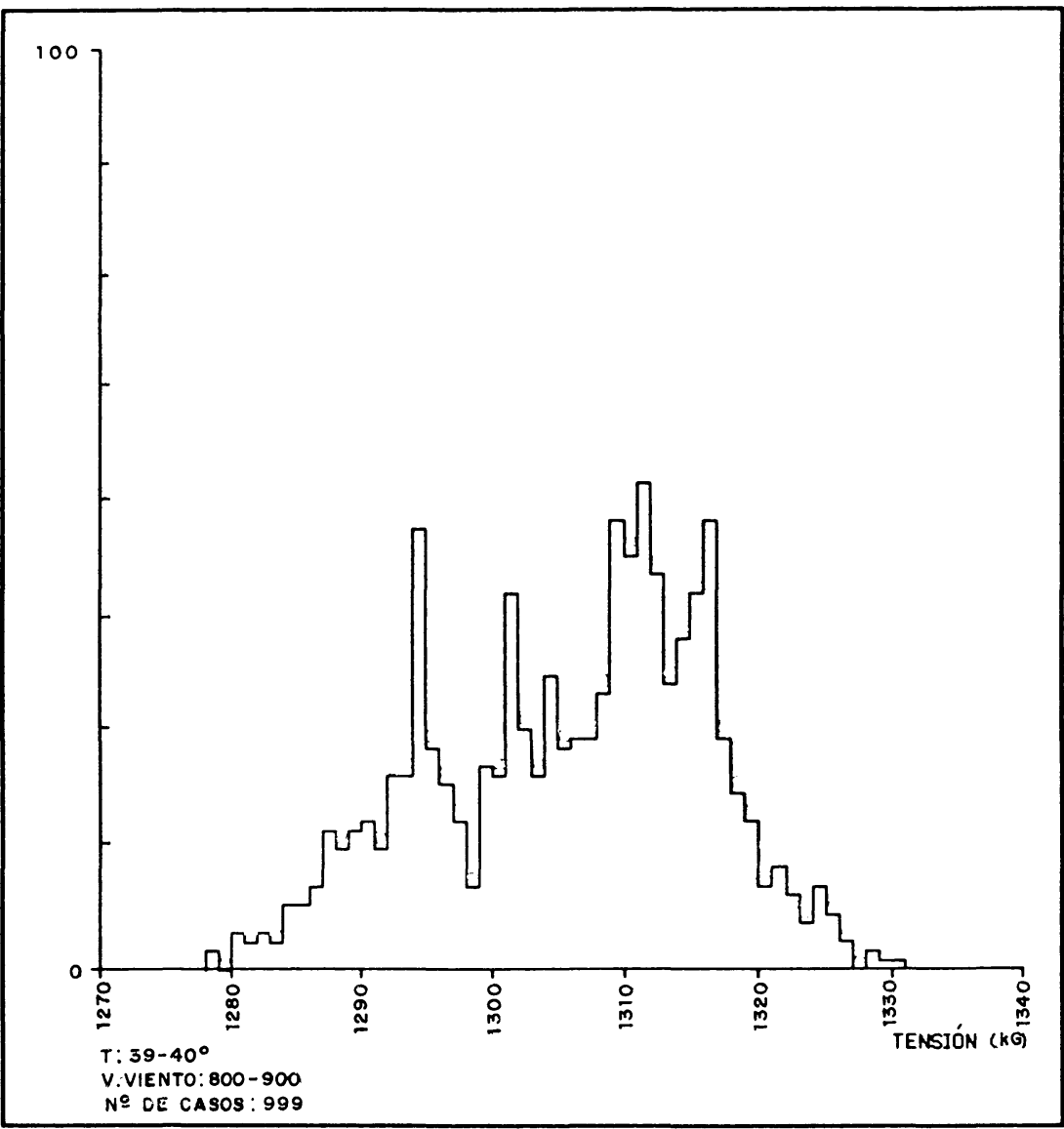

Fig. 15

Histograma de Tensiones. Cercha-1 cable inferior. $\mathrm{T}=39-40^{\circ}, \mathrm{VV}=8-9 \mathrm{~m} / \mathrm{s}$. VALOR TEÓRICO: $1.392 \mathrm{~kg}$. 


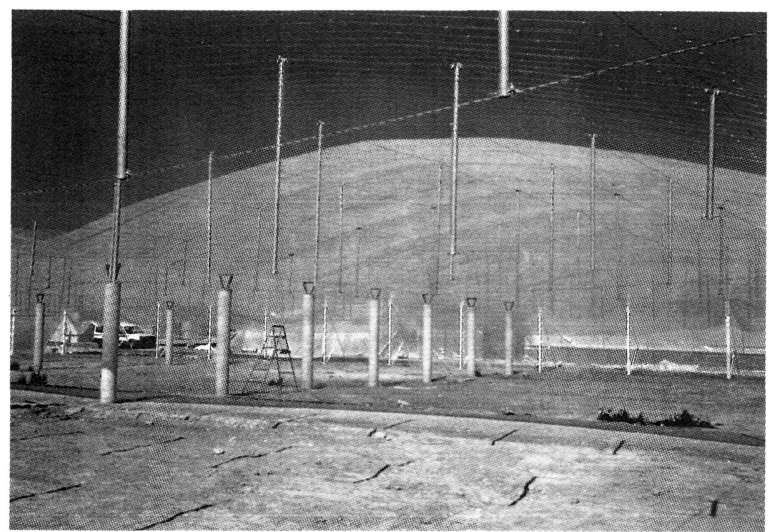

Fig. 16.- Cubierta plástica extendida parcialmente.

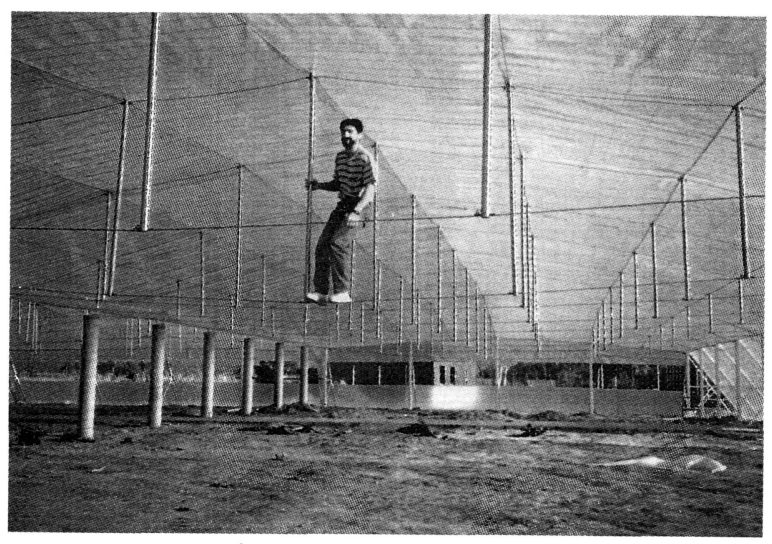

Fig. 17.- Una nave totalmente diáfana.

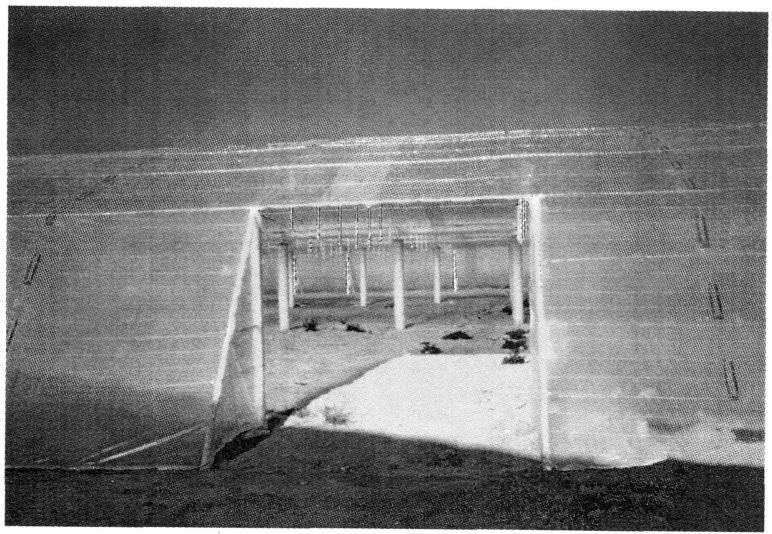

Fig. 18.- Vista interior.

por ejemplo para árboles, con incremento mínimo de costes y sin necesidad de modificar cadenas productivas.

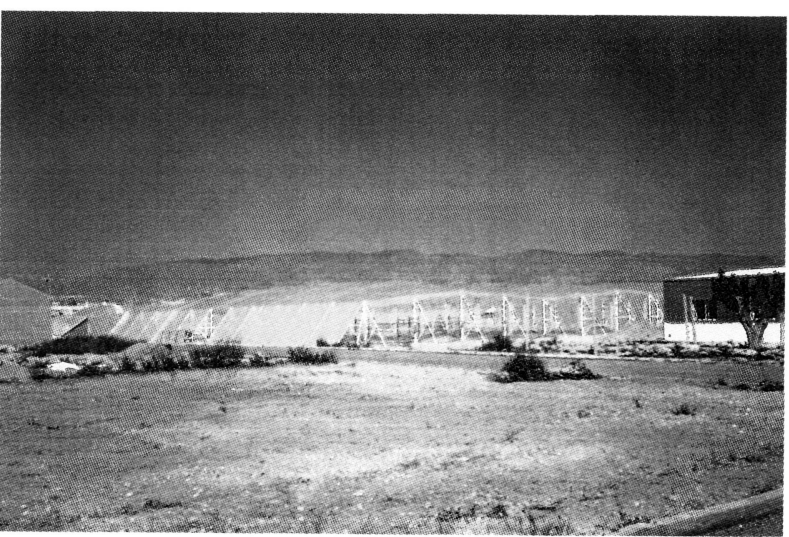

Fig. 19.- Panorámica tras el tendido del plástico.

- Su altura media, más elevada, proporciona mayor volumen. La creación en su interior (Fig. 18) de un gran colchón de aire, que actúa como amortiguador térmico, proporciona a la instalación una mayor inercia térmica.

\section{Diseño:}

- La forma de la cubierta optimiza la captación de la radiación solar, su homogeneidad y luminosidad (Fig. 19).

- El conjunto presenta un bajo coeficiente de forma lo que se traduce en un buen comportamiento frente al viento con coeficientes eólicos inferiores a 0,4 .

\section{EVALUACION ECONÓMICA}

Del análisis de los recursos empleados se deduce la siguiente:

Ecuación de coste de: (en términos de ejecución material)

$E M=P[3.855+0,46 h+v(691+H)]+S[0,236 h+423]$

EM: Ejecución Material (ptas)

P: $\quad$ Perímetro $(\mathrm{m})$

$\mathrm{v}$ : Volumen $\left(\mathrm{m}^{3}\right)$ de cimentación perimetral por $\mathrm{m}$ lineal

h: $\quad$ Precio horario mano de obra (ptas/hora)

$\mathrm{H}$ : $\quad$ Precio medio del $\mathrm{m}^{3}$ de hormigón (ptas $/ \mathrm{m}^{3}$ )

S: $\quad$ Superficie a cubrir $\left(\mathrm{m}^{2}\right)$

De ella se deduce, dado que trata de una estructura muy diáfana arriostrada fundamentalmente en el perímetro, la influencia de la relación perímetro/superficie de la parcela en el precio, por metro cuadrado de la instalación (Figura 20) 

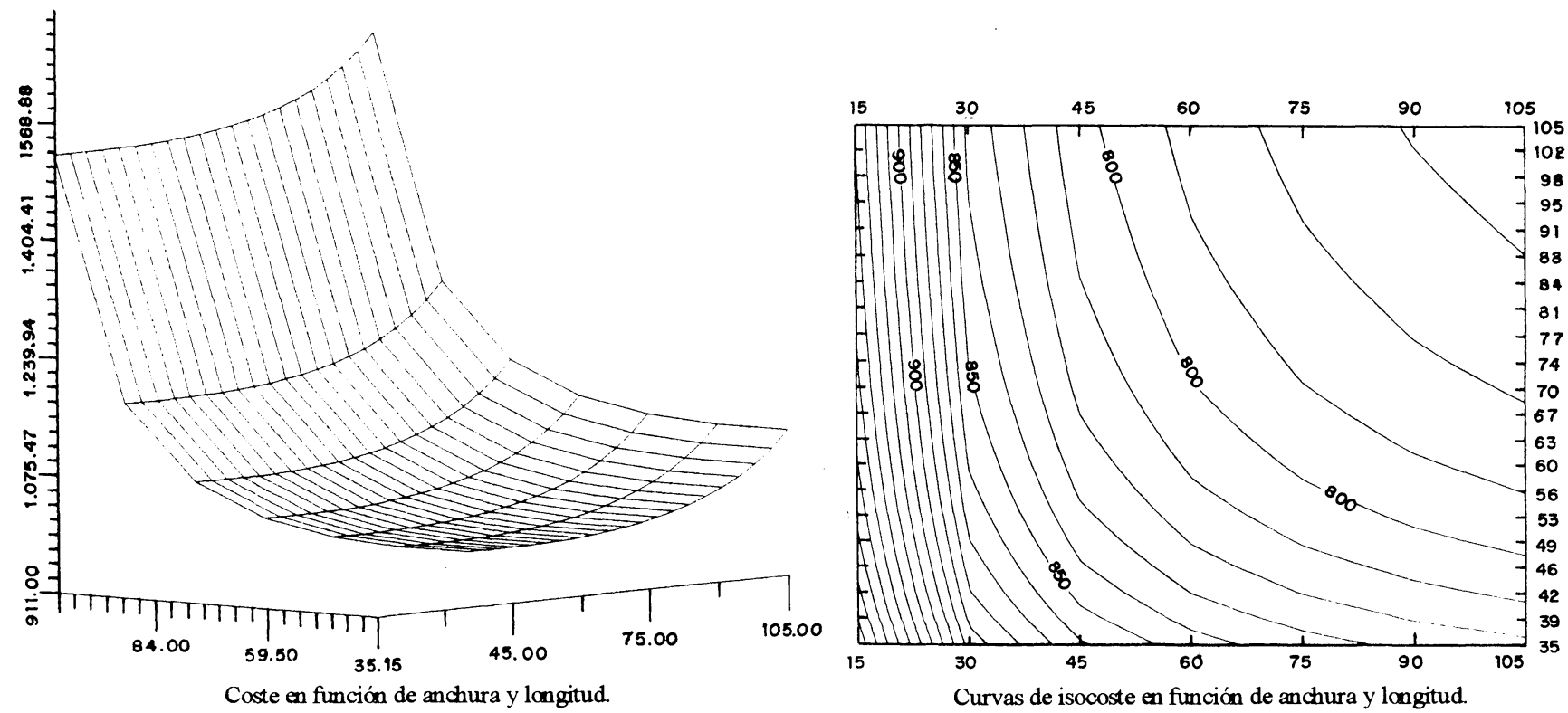

Fig. 20

\section{CONCLUSIONES}

- El modelo teórico desarrollado se presenta como una metodología adecuada para el cálculo de la estructura de cubierta, coincidiendo en gran medida los resultados obtenidos de éste con los datos experimentales.

- Los detalles constructivos diseñados han mostrado su funcionalidad en el montaje del prototipo y, junto al proceso de montaje ideado, han demostrado la viabilidad técnica de dicho prototipo.

- Se estima que los coeficientes eólicos sobre esta estructura flexible son menores que los establecidos por la NBE-AE-88 para superficies rígidas. Su ajuste necesitará de posteriores investigaciones. A este buen comportamiento frente a acciones de viento contribuye también su diseño aerodinámico

- Se han observado destensiones en algunos cables superiores, que si bien pueden corregirse con los tensores instalados, éstas deben evitarse con mayores tensiones iniciales en el montaje, del orden de $400 \mathrm{y}$ $1.000 \mathrm{kp}$ para los cables superior e inferior, respectivamente.

- En las tensiones definitivas influye el sentido de giro de las cerchas al pasar de la posición invertida a la posición normal, aumentando cuando se invierte el sentido de giro en dos cerchas consecutivas de la misma alineación.

- No se detectan influencias de la dirección del viento sobre las tensiones de la estructura, por lo que la orientación del invernadero sólo tiene que responder a la optimización de la captación de la radiación solar.

- Del estudio teórico con el modelo validado se deduce que existen elementos estructurales, postes centrales y laterales, que están sobredimensionados en el prototipo, pudiéndose reducir en los modelos comerciales

- La altura de los modelos comerciales debe elevarse hasta los $3 \mathrm{~m}$ en cabeza de pilares, lo que no entraña ninguna dificultad dadas las características de la estructura.

- El volumen de cimentación puede reducirse sensiblemente cuando mejore la calidad de los terrenos de cimentación.

- Del modelo teórico se deduce que la estructura de cubierta puede resistir las solicitaciones producidas por velocidades de viento de hasta $120 \mathrm{~km} / \mathrm{h}$ y las debidas a cargas gravitatorias de hasta $25 \mathrm{kp} / \mathrm{m}^{2}$. 